\title{
BMJ Open Systematic review on the primary and secondary reporting of the prevalence of ghostwriting in the medical literature
}

\author{
Serina Stretton
}

To cite: Stretton S. Systematic review on the primary and secondary reporting of the prevalence of ghostwriting in the medical literature. BMJ Open 2014;4:e004777.

doi:10.1136/bmjopen-2013004777

- Prepublication history for this paper is available online. To view these files please visit the journal online (http://dx.doi.org/10.1136/ bmjopen-2013-004777).

Received 2 January 2014 Revised 19 June 2014 Accepted 23 June 2014
CrossMark

ProScribe-Envision Pharma Group, Sydney, New South Wales, Australia

Correspondence to Dr Serina Stretton; serina.stretton@ EnvisionPharmaGroup.com

\section{ABSTRACT}

Background: Ghostwriting of industry-sponsored articles is unethical and is perceived to be common practice.

Objective: To systematically review how evidence for the prevalence of ghostwriting is reported in the medical literature.

Data sources: MEDLINE via PubMed 1966+, EMBASE 1966+, The Cochrane Library 1988+, Medical Writing 1998+, The American Medical Writers Association (AMWA) Journal 1986+, Council of Science Editors Annual Meetings 2007+, and the Peer Review Congress 1994+ were searched electronically (23 May 2013) using the search terms ghostwrit*, ghostauthor*, ghost AND writ*, ghost AND author*.

Eligibility criteria: All publication types were considered; only publications reporting a numerical estimate of possible ghostwriting prevalence were included.

Data extraction: Two independent reviewers screened the publications; discrepancies were resolved by consensus. Data to be collected included a numerical estimate of the prevalence of possible ghostwriting (primary outcome measure), definitions of ghostwriting reported, source of the reported prevalence, publication type and year, study design and sample population.

Results: Of the 848 publications retrieved and screened for eligibility, 48 reported numerical estimates for the prevalence of possible ghostwriting. Sixteen primary publications reported findings from cross-sectional surveys or descriptive analyses of published articles; 32 secondary publications cited published or unpublished evidence. Estimates on the prevalence of possible ghostwriting in primary and secondary publications varied markedly. Primary estimates were not suitable for meta-analysis because of the various definitions of ghostwriting used, study designs and types of populations or samples. Secondary estimates were not always reported or cited correctly or appropriately.

Conclusions: Evidence for the prevalence of ghostwriting in the medical literature is limited and can be outdated, misleading or mistaken. Researchers should not inflate estimates using non-standard definitions of ghostwriting nor conflate ghostwriting with other unethical authorship practices. Editors and peer reviewers should not accept articles that incorrectly cite or interpret primary publications that report the prevalence of ghostwriting.

\section{Strengths and limitations of this study}

- First systematic review on the reporting of the prevalence of ghostwriting in the medical literature.

- A broad search strategy was employed with few restrictions to minimise any potential for publication or language bias; there were no restrictions on language and all study designs and publication types were considered.

- Study heterogeneity in the outcomes reported and populations investigated precluded synthesis of the data.

- The retrospective and self-reported nature of the data collection increased the risk of selection bias in the studies.

- Most included studies were not broadly representative of the peer-reviewed medical literature.

\section{INTRODUCTION}

Ghostwriting occurs when paid or unpaid writing contributions to a manuscript that do not meet authorship criteria are not disclosed in the acknowledgments. ${ }^{1}$ This practice is considered to be distinct from ghost authoring, where contributions to a manuscript that do merit authorship are not disclosed in the author byline. ${ }^{1}$ However, part of the challenge in understanding the prevalence of ghostwriting is the current confusion surrounding these unethical practices. For example, the widely used Council of Science Editors definition of ghost authors (ie, individuals who participate in research, data analysis and/or writing of a manuscript but are not named or disclosed in the author byline or acknowledgments) does not distinguish between ghost authors and ghostwriters. ${ }^{2}$

Ghostwriting of peer-reviewed journal publications in the medical literature is believed to be common practice. ${ }^{3-5}$ This belief is supported, to a large extent, by highly publicised cases, primarily from the 1990s and early 2000 s, of pharmaceutical companies and authors who had used ghostwriters to prepare manuscripts for publication in 
medical journals. ${ }^{6-8}$ Such cases are highly unethical because the role of the commercial sponsor and any other potential conflicts of interest were hidden. Estimating the prevalence of ghostwriting in the medical literature has been hindered not only by the different definitions of ghostwriting and ghost authoring, but also by the failure of authors to distinguish ghostwriting, which is unethical, from professional medical writing support, which is ethical. ${ }^{9}{ }^{10}$ Ghostwriters keep their involvement in a manuscript hidden, whereas professional medical writers disclose their involvement and follow ethical publication practices. ${ }^{19-11}$ Consistent with the authorship criteria recommended by the International Committee of Medical Journal Editors (ICMJE), professional medical writers who provide writing assistance and do not meet all of the ICMJE authorship criteria should be acknowledged rather than listed as authors. ${ }^{12}$ The prevalence of disclosed professional medical writing assistance in medical journals has been estimated to be between $6 \%$ and $11 \%^{10} 13$ and the legitimate value that this medical writing assistance can bring to improving the quality, timeliness and integrity of reporting in medical journals has been demonstrated. ${ }^{1} 14 \quad 15$ However, the exact prevalence of ghostwriting and other forms of undisclosed contributions to papers published in medical journals is unknown.

The primary objective of this study was to conduct the first systematic review on how the prevalence of ghostwriting is reported in the medical literature. The secondary objectives were to assess the variability of the reported estimates of the prevalence of ghostwriting and investigate the source for these estimates.

\section{MATERIALS AND METHODS}

\section{Literature search strategy}

A search strategy was developed to retrieve publications from the medical literature that reported quantitative estimates of the prevalence of ghostwriting. The following databases were searched on 23 May 2013: MEDLINE via PubMed (1966+); EMBASE (1966+); The Cochrane Library, including the Cochrane Database of Systematic Reviews and Cochrane Central Register of Controlled Trials (1988+); The AMWA Journal (1986+, journal of the American Medical Writers Association); Medical Writing (1998+, journal of the European Medical Writers Association, formerly known as The Write Stuff); Council of Science Editors annual meetings (2007+); and the International Congress on Peer Review and Biomedical Publication meetings (1994+). General text was searched using the following search terms: ghostwrit*, ghostauthor*, ghost AND writ*, ghost AND author*. Truncation symbols and Boolean operators (AND, OR) were used wherever possible.

Two reviewers (SS, Sericka McGee) independently screened the title and abstracts of all retrieved publications using prespecified eligibility criteria; the full text of potentially eligible publications was screened to confirm eligibility for inclusion. Any discrepancies between the reviewers were resolved by consensus. The reference lists of relevant reviews and other publications were screened by hand to identify any additional publications for inclusion.

\section{Eligibility criteria}

The eligibility criteria were prespecified. Publications were included if they reported a numerical estimate of the prevalence of possible ghostwriting. Publications were excluded if they were duplicate publications from different databases or abstracts of subsequently published full-text articles, did not report any outcomes related to ghostwriting or reported qualitative estimate (s) of the prevalence of possible ghostwriting. No restrictions on language were included in the eligibility criteria. If needed, the abstracts of publications written in non-English language were to be translated to assist in screening for eligibility. If publications were eligible for inclusion, the full texts of the publications were to be translated to English.

\section{Data extraction and analysis}

The primary and secondary outcomes and data to be collected were prespecified. Data to be collected included publication type and year, study design and sample population, definitions of ghostwriting reported, reported prevalence of possible ghostwriting and the source of the reported prevalence of ghostwriting. The prevalence of possible ghostwriting was reported as published. No unpublished data from the retrieved literature were reported and values for prevalence were not extrapolated from published data.

The primary outcome measure for the systematic review was a numerical estimate of the prevalence of possible ghostwriting. The reported definitions of possible ghostwriting were categorised according to the following standard definitions, irrespective of the term used to describe the practice in the publication. Undisclosed writing contributions to a manuscript were defined as: (1) ghostwriting if they were described as not meriting authorship and were not listed in the acknowledgments; ${ }^{916}$ and (2) ghost authoring if they did merit authorship and were not listed in the author byline. ${ }^{1}$ Consistent with recommendations from international medical journal editors, ${ }^{12}{ }^{14}$ Good Publication Practice guidelines $^{11}$ and professional medical writing associations, ${ }^{17} 18$ disclosed writing contributions to a manuscript that did not merit authorship and were disclosed in the acknowledgments were not categorised as ghostwriting.

The key factors considered when assessing study quality were study design, the population assessed and how ghostwriting was defined. While there is no consensus on the best practice for reporting survey research, ${ }^{19}$ the quality of the cross-sectional surveys was determined by an assessment of the following factors: validation or pretesting of the survey questions, anonymity of the response, sample size and response rate. 


\section{RESULTS}

\section{Publication selection}

A total of 848 articles were retrieved from the literature search, 800 were excluded and 48 met the eligibility criteria and were included in the systematic review (figure 1). The main reasons for exclusion were publications not relevant to ghostwriting $(n=539)$, duplicate publications from different databases $(n=129)$ and publications not reporting a numerical estimate of the prevalence of possible ghostwriting $(n=124)$. The titles or abstracts of the eight publications that were excluded because the full text or abstract could not be retrieved were reviewed; none were considered to report a numerical estimate of the prevalence of possible ghostwriting. Overall, eligible publications included 16 primary publications that reported original research on the prevalence of possible ghostwriting and 32 secondary publications that cited published or unpublished evidence of possible ghostwriting.

\section{Primary publications}

Publication characteristics

Of the 16 primary publications (table 1 ), there were 13 full-text publications ${ }^{6} \quad 8 \quad 20-30$ and three conference abstracts $^{31-33}$ that reported a numerical estimate of possible ghostwriting. Twelve publications ${ }^{20-28} 31-33$ reported findings from cross-sectional surveys and four publications $^{6} 82930$ reported findings from descriptive analyses of published articles. Most cross-sectional surveys were reasonably well reported. The surveys used were not validated but most included pretested questions, required an anonymous response, were conducted in targeted populations (ie, all individuals invited to participate

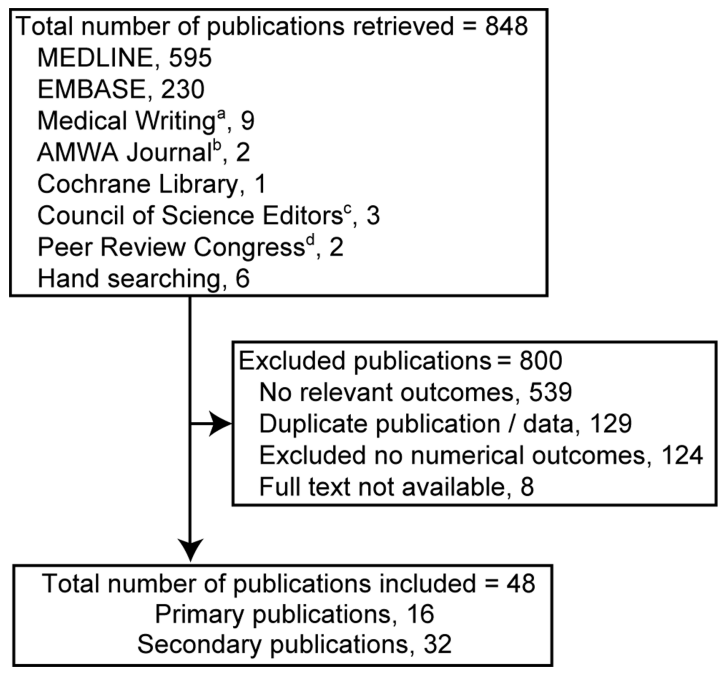

Figure 1 Selection of publications reporting a numerical estimate of the prevalence of ghostwriting. Electronic databases were searched on 23 May 2013. ${ }^{a}$ Journal of the European Medical Writers Association, formerly known as The Write Stuff. ${ }^{\mathrm{b}}$ Journal of the American Medical Writers Association. ${ }^{\mathrm{C}}$ Council of Science Editors Annual Meetings. ${ }^{d}$ International Congress on Peer Review and Biomedical Publication. were involved in the preparation of peer-reviewed manuscripts), and reported a sample size and response rate. Of the cross-sectional surveys conducted in targeted populations (eg, corresponding or first authors), six had response rates greater than $50 \%$, two had response rates less than $50 \%$, and one did not report a response rate (table 1). Of the cross-sectional surveys conducted in non-targeted populations (eg, individuals invited to participate who may or may not have been involved in providing medical writing assistance for peer-reviewed publications), three had low (12\% to $28 \%$ ) response rates and one did not report a response rate (table 1).

Not all cross-sectional surveys were broadly representative of the peer-reviewed medical literature. The cross-sectional surveys were conducted in single populations of academic staff or healthcare professionals, ${ }^{22}{ }^{31}$ members of medical writing associations, ${ }^{25} 33$ or corresponding or first authors. The corresponding or first authors were surveyed from single journals (Cochrane reviews, Chinese Medical Journal, Archives of Iranian Medicine), ${ }^{23}{ }^{26}{ }^{32}$ two Danish journals, ${ }^{21}$ three Iranian journals, ${ }^{24}$ and three pharmacy journals. ${ }^{28}$ Two cross-sectional surveys of corresponding authors were conducted in six general medicine journals. ${ }^{20} 27$

The descriptive analyses of published articles were conducted in single populations and included an analysis of sertraline publications from 1998 to $2000,{ }^{6}$ rofecoxib reviews from 1996 to $2004,{ }^{8}$ publications from industry-initiated trials in Denmark from 1994 to $1995,{ }^{30}$ and from published non-inferiority trials from 1989 to $2009 .^{29}$

\section{Reported prevalence of ghostwriting}

The reported prevalence of possible ghostwriting in the primary publications varied markedly and was not suitable for meta-analysis because of the different populations assessed, the different methods used to generate the estimates and the various definitions that were used (tables 1 and 2). All cross-sectional surveys reported a definition of ghostwriting or ghost authoring, but most definitions did not differentiate contributions that merited authorship from those that did not merit authorship (table 2).

Four cross-sectional surveys reported the prevalence of ghostwriting where the definition provided could be categorised as undisclosed contributions that do not merit authorship. ${ }^{20} 252733$ Findings from these surveys, which were repeated at different time points, suggested that the prevalence of ghostwriting is low and decreasing. Flanagin ${ }^{27}$ and Wislar ${ }^{20}$ conducted two similar crosssectional surveys of corresponding authors of articles published in six general medicine journals in $1996^{27}$ and in $2008 .^{20}$ The surveys, which included a core set of three journals (Annals of Internal Medicine, JAMA, the New England Journal of Medicine) at each time point and in targeted populations, used pretested questions that required an anonymous response and had response rates greater than $65 \%$. The prevalence of ghost authoring (which included ghostwriting) was estimated to be $11.5 \%$ in 1996 and $7.9 \%$ in 2008. The prevalence of 
Table 1 Characteristics of primary publications reporting original evidence of ghostwriting

\begin{tabular}{|c|c|c|c|c|c|}
\hline Publication & Population & Survey description & $\begin{array}{l}\text { Data } \\
\text { collected }\end{array}$ & $\mathbf{N}$ & $\mathbf{R R}$ \\
\hline \multicolumn{6}{|c|}{ Cross-sectional surveys-authors or corresponding authors } \\
\hline Flanagin et $a P^{7}$ & Six general medicine journals* & $\begin{array}{l}\text { Self-administered, postal, anonymous, pretested, } \\
\text { targeted }\end{array}$ & 1996 & 809 & $69 \%$ \\
\hline Mowatt et $a f^{23}$ & Published Cochrane reviews & $\begin{array}{l}\text { Self-administered, online, anonymous, pretested, } \\
\text { targeted }\end{array}$ & 1999 & 362 & $63 \%$ \\
\hline Hao et $a \beta^{\beta 2}$ (Abstract) & Chin Med J & Self-administered, email, not anonymous, targeted & $2008 \dagger$ & 220 & $86 \%$ \\
\hline Dotson and Slaughter ${ }^{28}$ & Three pharmacy journals & $\begin{array}{l}\text { Self-administered, online, anonymous, pretested, } \\
\text { targeted }\end{array}$ & 2009 & 112 & $25 \%$ \\
\hline Mirzazadeh et $a^{4}$ & Three Iranian journals & Self-administered, email, not anonymous, targeted & 2009-2010 & NR & NR \\
\hline Wislar et a ${ }^{20}$ & Six general medicine journalsł & $\begin{array}{l}\text { Self-administered, online, anonymous, pretested, } \\
\text { targeted }\end{array}$ & 2008 & 622 & $70 \%$ \\
\hline Ghajarzadeh ${ }^{26}$ & Arch Iran Med (based on student theses) & Self-administered, email, anonymous, targeted & 2005-2007 & 30 & $49 \%$ \\
\hline Vinther and Rosenberg ${ }^{21}$ & Ugeskr Laeger and Dan Med J & $\begin{array}{l}\text { Self-administered, online, anonymous, pretested, } \\
\text { targeted }\end{array}$ & 2010 & 272 & $62 \%$ \\
\hline \multicolumn{6}{|c|}{ Cross-sectional surveys-healthcare professionals } \\
\hline Price et $a R^{2}$ & Health academic staff & $\begin{array}{l}\text { Self-administered, postal, anonymous, pretested, } \\
\text { targeted }\end{array}$ & NR & 166 & $59 \%$ \\
\hline Rees et $a{ }^{\beta 1}$ (Abstract) & Registered users of EPG Online & Self-administered, online, anonymous, non-targeted & NR & 295 & NR \\
\hline \multicolumn{6}{|c|}{ Cross-sectional surveys-members of medical writing associations } \\
\hline \multirow[t]{2}{*}{ Jacobs and Hamilton 25} & \multirow[t]{2}{*}{ EMWA/AMWA members } & \multirow{2}{*}{$\begin{array}{l}\text { Self-administered, online, anonymous, pretested, } \\
\text { non-targeted }\end{array}$} & 2005 & 843 & $28 \%$ \\
\hline & & & 2008 & 773 & $14 \%$ \\
\hline $\begin{array}{l}\text { Hamilton and Jacobs } \\
2012^{33} \text { (Abstract) }\end{array}$ & EMWA/AMWA members & $\begin{array}{l}\text { Self-administered, online, anonymous, pretested, } \\
\text { non-targeted }\end{array}$ & 2011 & 620 & $\begin{array}{l}12 \% \\
\S\end{array}$ \\
\hline \multicolumn{6}{|c|}{ Publication reviews and descriptive analysis } \\
\hline Healy and Cattell ${ }^{6}$ & Articles on sertraline & NA & 1998-2000 & 96 & NA \\
\hline Gøtzsche et $a \beta^{\beta 0}$ & $\begin{array}{l}\text { Articles on Danish industry-initiated trials approved } \\
1994-1995\end{array}$ & NA & NR & 44 & NA \\
\hline Ross et $a \beta^{\beta}$ & Reviews on rofecoxib associated with Merck support & NA & 1996-2004 & 72 & NA \\
\hline Suda et $a$ f $^{9}$ & Non-inferiority clinical trials & NA & 1989-2009 & 583 & NA \\
\hline
\end{tabular}


Table 2 Primary publications reporting estimates of the prevalence of possible ghostwriting

\section{Publication} Measure of possible ghostwriting reported by authors

estimate \% (n)

Cross-sectional surveys reporting ghostwriting

Flanagin et $a^{7}$

Wislar et a ${ }^{20}$

Unnamed individual who participated in the writing

Jacobs and Hamilton, Hamilton Undisclosed medical writing assistance not qualifying for authorship

and Jacobs 2533

Cross-sectional surveys reporting combined ghost authoring and ghostwriting

Flanagin et a ${ }^{77}$

Price et $a^{22}$

Mowatt et al ${ }^{23}$

Hao et $a^{\beta 2}$

Dotson and Slaughter 28

Wislar et afo

Failure to name, as an author, individuals who made substantial contributions to the research or writing or an unidentified medical writer

Failure to name, as an author, individuals who made substantial contributions to the research or writing Individual merited authorship or had assisted with drafting but not listed as an author or acknowledged English-language speakers assisted with writing but not identified as authors or acknowledged

Failure to name, as an author, individuals who made substantial contributions to the research or writing Failure to name, as an author, individuals who made substantial contributions to the research or writing of the article or an unnamed individual who participated in the writing

Cross-sectional surveys reporting ghost authoring

Mirzazadeh et aR

Ghajarzadeh 26

Failure to name, as an author, individuals who made substantial contributions to the research

Vinther and Rosenberg ${ }^{21}$

Failure to name, as an author, students who made substantial contributions to the research $\neq$

Individual merited authorship but not listed as an author

Individual merited authorship but not listed as an author

Publication reviews and descriptive analysis reporting possible ghost authoring or ghostwriting

Healy and Cattell ${ }^{6}$

Published articles coordinated by a medical information company, including acknowledged medical writing support§

Gøtzsche et $a{ }^{\beta 0}$

Individuals who wrote the trial protocol, conducted the statistical analyses or wrote the manuscript but were not listed as authors, not members of a study group or steering committee or not disclosed in an

acknowledgment

Ross et $a \beta$

Published reviews associated with Merck support and with a single external authorq
$1.4 \%(11 / 809)$ of articles $0.2 \%(1 / 622)$ of articles* 2005: $61.8 \%(N R)$

2009: $41.7 \%(N R)$

2011: $33.0 \%(N R)$ of articles†

$11.5 \%(93 / 809)$ of articles

$24.1 \%(40 / 166)$ of authors $8.8 \%(32 / 362)$ of articles $10.4 \%$ (NR) of authors $0.9 \%(1 / 112)$ of articles $7.9 \%(49 / 622)$ of articles $21.4 \%(25 / \mathrm{NR})$ of authors $0.7 \%(2 / 296)$ of articles $2.4 \%(6 / 245)$ of articles $70 \%$ (NR/202) of published authors

$57.3 \%(55 / 96)$ of articles

$75.0 \%(33 / 44)$ of trials $69.4 \%(50 / 72)$ of reviews

${ }^{*}$ Available as online supplementary data.

†Values represent the mean weighted percentage of publications that were ghostwritten by respondents. Findings were weighted in proportion to the number of manuscripts the respondent wrote per year.

†Students were classified as ghostwriters if the student was not named as an author and if the results reported in the publications were based on the results of their theses.

$\S$ Authors conclude data provide quantification of the possible extent of ghostwriting based on a single drug. Of the 55 published articles that were coordinated through a medical information company, 2 included medical writing assistance that was acknowledged in the published article.

IPublished review articles had been identified from correspondence between Merck and a medical publishing company, from Merck publication status reports, or were affiliated with an author named within the correspondence or publication status reports. The authors did not report whether medical writing assistance was acknowledged in the published article.

NR, not reported. 
ghostwriting from these surveys was estimated to be $1.4 \%$ in 1996 and $0.2 \%$ in 2008. Hamilton and Jacobs conducted a cross-sectional survey of members of two medical writing associations in 2005, 2008 and 2011..$^{25} 33$ The survey, which was not conducted in targeted populations, used pretested questions that required an anonymous response and had response rates from $12 \%$ to $28 \%$ of the total population surveyed (ie, not all participants surveyed were involved in the preparation of peerreviewed manuscripts). Findings from these surveys showed that the mean weighted percentage of publications that were ghostwritten by respondents had decreased from $61.8 \%$ in 2005 to $33 \%$ in 2011. Findings were weighted in proportion to the number of manuscripts the respondent wrote per year.

Six cross-sectional survey publications reported the prevalence of possible ghostwriting (ie, the prevalence of ghostwriting and ghost authoring combined). ${ }^{20} 2223272832$ The prevalence of possible ghostwriting reported in these cross-sectional surveys varied from $0.9 \%$ to $24.1 \%$ of publications or authors.

Four cross-sectional survey publications reported the prevalence of ghost authoring. ${ }^{21} 242631$ The prevalence of ghost authoring reported in these cross-sectional surveys varied from $0.7 \%$ to $70 \%$ of publications or authors.

The descriptive analyses of published articles on sertraline and rofecoxib did not include a prespecified definition of ghost authoring or ghostwriting ${ }^{6}{ }^{8}$; in these studies, possible ghostwriting or ghost authoring was assumed in publications associated with industrysponsored support (table 2). The descriptive analysis of industry-initiated trials in Denmark used a non-standard definition of ghost authors; in this study ghost authors were defined as individuals, not named as authors, who were involved in writing the protocol, conducting the statistical analyses or writing the manuscript. ${ }^{30}$ The prevalence of possible ghostwriting in the two descriptive analyses of single drugs that did not include a prespecified definition of ghostwriting was $57 \%$ of articles on sertraline published from 1998 to $2000^{6}$ and $69 \%$ of reviews on rofecoxib published from 1996 to $2004 .^{8}$ The prevalence of possible ghostwriting in the descriptive analysis of Danish industry-initiated trials, which used a nonstandard definition of authorship, was $75 \%$ of Danish initiated trials approved in 1994 to $1995 .{ }^{30}$ The descriptive analysis of non-inferiority trials ${ }^{29}$ was not considered further as the definition of ghostwriting used was consistent with disclosed medical writing assistance. In this study ghostwriters were defined as acknowledged individuals, other than authors, who contributed to the writing and were affiliated with an industry-sponsored study. ${ }^{29}$ The prevalence of disclosed medical writing assistance in this descriptive analysis was $17.3 \%$ (101/583) of clinical trials.

\section{Secondary publications}

Publication characteristics

Of the 32 secondary publications, there were 13 review articles, 10 editorials, 5 commentaries, 3 news articles and 1 government report, with most being published after 2008 (table 3). Most publications cited primary sources as evidence of the prevalence of possible ghostwriting (table 3), with the cited prevalence of possible ghostwriting varying from $6 \%$ of publications to $100 \%$ of publications involving drugs. In most secondary publications, the information on the prevalence of ghostwriting was not reported consistently compared with the cited evidence (table 3). Misleading and mistaken information was reported in many publications that: (1) did not distinguish between ghostwriting and ghost authoring; (2) included acknowledged medical writing assistance or a combined estimate of guest authorship and ghost authorship as ghostwriting; (3) generalised findings from publication reviews and analyses of specific data sets to wider populations of publications or industrysponsored trials; or (4) cited personal communications or informal surveys where the original source was unpublished and could not be verified (table 3 ).

\section{Case study of misleading and mistaken evidence}

An analysis of secondary publications citing the possible prevalence of ghostwriting in sertraline publications provides an illuminating case study on how misleading or mistaken evidence can enter and remain in the medical literature. In 2003, Healy and Cattell ${ }^{6}$ showed that $57 \%$ (55/96) of articles on sertraline published from 1998 to 2000 were coordinated through a medical information company and only two acknowledged medical writing assistance. The authors concluded that these data provided information on the 'possible extent of ghostwriting based on a single drug'. On October 14, 2004, Healy also provided evidence on the influence of the pharmaceutical industry on key groups at a UK House of Commons Health Select Committee investigation. ${ }^{62}$ In answer to Question 197, Healy stated the following:

My estimate is that, even in journals like the BMJ, the Lancet, the New England Journal of Medicine and JAMA, the leading journals in the field, if these articles have to do with therapeutics, with drugs, it may be worse perhaps for psychiatry than elsewhere, but I doubt it, $50 \%$ of these articles are ghostwritten. It may be higher.

Healy and Cattell's original evidence has been cited incorrectly and interchangeably with Healy's statement to the House of Commons Health Select Committee investigation (figure 2). Although findings from the primary publication have been cited and interpreted correctly in two secondary publications, ${ }^{34} 35$ many secondary publications incorrectly cited Healy's original evidence and statement to the House of Commons Health Select Committee investigation (figure 2).

\section{DISCUSSION}

This systematic review on the reported prevalence of ghostwriting in the medical literature showed that the estimates of the prevalence of ghostwriting in primary 
Table 3 Characteristics of secondary publications citing evidence of possible ghostwriting

\begin{tabular}{|c|c|c|c|}
\hline Publication & Cited evidence & $\begin{array}{l}\text { Consistent with } \\
\text { cited source(s) }\end{array}$ & Comment \\
\hline Elliot $^{34}$ & Primary & No & Did not distinguish GW from GA \\
\hline Moffat and Elliott ${ }^{35}$ & Primary & No & Did not distinguish $\mathrm{GW}$ from $\mathrm{GA}$ \\
\hline Schiefe $e^{36}$ & Primary & No & Did not distinguish GW from GA \\
\hline MacLennan et $a \beta^{\beta 7}$ & Primary & No & Did not distinguish GW from GA \\
\hline Nahai $^{38}$ & Secondary & No & Did not distinguish GW from GA \\
\hline $\mathrm{Ngai}^{39}$ & Primary & Yes & Did not distinguish GW from GA \\
\hline Bosch $^{40}$ & Primary & Yes & Did not distinguish GW from GA \\
\hline Wiwanitkit $^{41}$ & Primary & No & $\begin{array}{l}\text { Did not distinguish GW from GA or GA from guest } \\
\text { authorship }\end{array}$ \\
\hline Krimsky $^{42}$ & Primary & No & $\begin{array}{l}\text { Did not distinguish GW from GA or GA from guest } \\
\text { authorship }\end{array}$ \\
\hline Langdon-Neuner $2008^{43}$ & $\begin{array}{l}\text { Primary and } \\
\text { secondary }\end{array}$ & No & $\begin{array}{l}\text { Did not distinguish GW from GA or GW from disclosed } \\
\text { medical writing support }\end{array}$ \\
\hline Tharyan $^{44}$ & Primary & Yes & $\begin{array}{l}\text { Did not distinguish GW from GA or GW from disclosed } \\
\text { medical writing assistance }\end{array}$ \\
\hline Paul $^{45}$ & Primary & Yes & $\begin{array}{l}\text { Did not distinguish GW from GA from disclosed medical } \\
\text { writing assistance }\end{array}$ \\
\hline Bavdekar $^{46}$ & Primary & No & $\begin{array}{l}\text { Did not distinguish GW from GA and generalised } \\
\text { evidence to a wider population }\end{array}$ \\
\hline Górski and Letkiewicz ${ }^{47}$ & $\begin{array}{l}\text { Primary and } \\
\text { secondary }\end{array}$ & No & $\begin{array}{l}\text { Did not distinguish GW from GA and generalised } \\
\text { evidence to a wider population }\end{array}$ \\
\hline $\begin{array}{l}\text { Matias-Guiu and } \\
\text { García-Ramos }^{48}\end{array}$ & $\begin{array}{l}\text { Primary and } \\
\text { secondary }\end{array}$ & No & $\begin{array}{l}\text { Did not distinguish GW from GA and generalised } \\
\text { evidence to a wider population }\end{array}$ \\
\hline McHenry ${ }^{49}$ & Gov. report & No & Generalised evidence to a wider population \\
\hline Healy ${ }^{50}$ & $\begin{array}{l}\text { Primary and } \\
\text { secondary }\end{array}$ & No/ND & $\begin{array}{l}\text { Generalised evidence to a wider population and reported } \\
\text { personal opinion of GW prevalence }\end{array}$ \\
\hline Abbasi $^{4}$ & Secondary & No & Secondary publication cited named individual \\
\hline Mitrany ${ }^{51}$ & None & ND & Cited named individual \\
\hline Collier ${ }^{5}$ & None & ND & Cited named individual \\
\hline Kmietowicz ${ }^{52}$ & None & ND & Cited named individual \\
\hline Matthews ${ }^{3}$ & None & ND & Cited unpublished data \\
\hline Bonita et $a^{53}$ & Primary & Yes & Consistent with cited source \\
\hline Anon $2011^{54}$ & Primary & Yes & Consistent with cited source \\
\hline Anon $2007^{55}$ & Primary & Yes & Consistent with cited source \\
\hline Editors $^{15}$ & Primary & Yes & Consistent with cited source \\
\hline Baethge $^{56}$ & Primary & Yes & Consistent with cited source \\
\hline Flanagin $2010^{57}$ & Primary & Yes & Consistent with cited source \\
\hline Murray et $a^{58}$ & Primary & Yes & Consistent with cited source \\
\hline Moore 59 & Secondary & Yes & Consistent with cited source \\
\hline Hargreaves ${ }^{60}$ & Primary & Yes & Consistent with cited source \\
\hline Jones $^{61}$ & Primary & Yes & Consistent with cited source \\
\hline
\end{tabular}

CSE, Council of Science Editors; GA, ghost authoring; Gov, government; GW, ghostwriting; ND, not determined.

publications varied markedly and were influenced by the definitions used, the types of study designs and the type of population or sample assessed. In addition, secondary publications often cited outdated, misleading or mistaken evidence of the reported prevalence of ghostwriting, with many publications not distinguishing ghostwriting from ghost authoring.

Although evidence from descriptive analyses can highlight the extent of ghostwriting in single populations, evidence from well-conducted cross-sectional surveys have the potential to provide estimates of the prevalence of ghostwriting that may be generalised to the majority of peer-reviewed publications. Despite this, many of the cross-sectional surveys retrieved in this systematic review were conducted in limited populations that were not broadly representative of the peer-reviewed medical literature. In addition, many of the cross-sectional surveys did not differentiate between contributions that merited authorship from those that did not merit authorship and provided, at best, an estimate of possible ghostwriting. The reported prevalence of ghostwriting, where ghostwriting was defined as undisclosed contributions that did not merit authorship, was retrieved from the two cross-sectional surveys of corresponding authors 


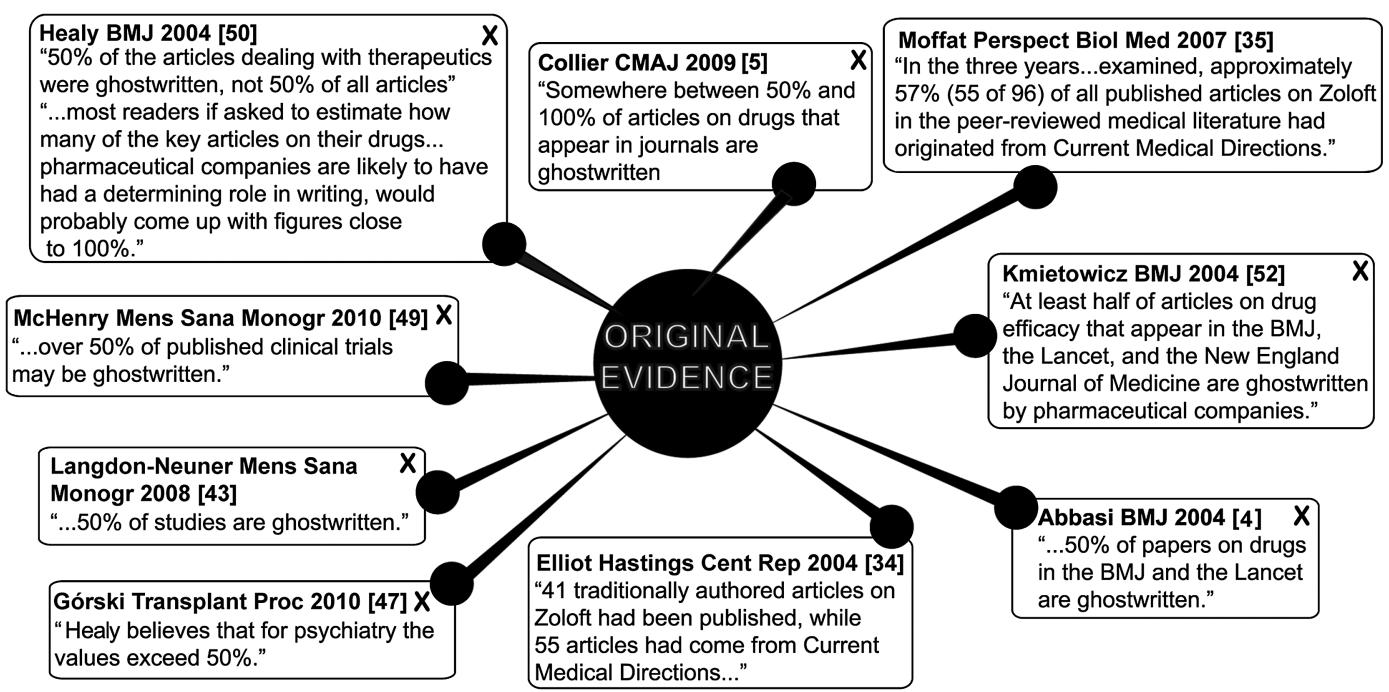

Figure 2 Case study of original versus cited evidence of ghostwriting prevalence. Contrast between original evidence on the prevalence of possible ghostwriting in sertraline publications from 1998 to 2000 and the subsequent citations of this evidence. Source of the original published evidence: Healy and Cattell ${ }^{6}$ showed that 55 of $96(57 \%)$ articles published on sertraline from 1998 to 2000 were coordinated through a medical communications company and concluded that these data provided information on the 'possible extent of ghostwriting based on a single drug'. Inaccurate reporting from the cited source is marked with a cross.

from several general medicine journals and by crosssectional surveys (repeated on three separate occasions from 2005 to 2011) of members of two major medical writing associations. Together the findings from these surveys suggested that the prevalence of ghostwriting has decreased in recent years. However, while the findings from these surveys may be considered more broadly representative of the peer-reviewed medical literature than surveys focused on single journals, single countries or single subject areas, interpretation of these findings should take into account that respondents were required to retrospectively self-report potentially unethical or unprofessional behaviour.

Unethical authorship practices are a major concern and are an increasingly recognised problem in the medical literature. ${ }^{63}$ As the findings from this systematic review suggest, some of these perceived problems may arise from the considerable, but unnecessary, confusion and disagreement surrounding the definitions of ghostwriting. As recognised by the World Association of Medical Editors, professional medical writers can have a legitimate role in assisting authors to communicate their research findings in the peer-reviewed literature. ${ }^{14}$ Professional medical writers can and do improve the timeliness and quality of reporting and can assist investigators and industry sponsors to meet their ethical commitments to the disclosure and publishing of clinical trial results. ${ }^{164-66}$ As such, the misleading and mistaken reporting of the prevalence of ghostwriting that was evident in the secondary publications retrieved in this systematic review is disappointing. Specifically, the confusion surrounding definitions of ghost authorship and ghostwriting and the unbalanced focus on industry as the source of unethical authorship practices in the secondary publications takes attention away from the need to focus on all types of unethical contributions in peerreviewed publications, irrespective of the source of the unethical practice.

The various definitions of ghostwriting and ghost authoring found in this systematic review highlight the considerable disagreement in the medical literature with regard to the definitions of ghostwriting and ghost authoring and whether a distinction should be made between these unethical practices. Although the Council of Science Editors uses a straightforward and broad definition of ghost authors that does not distinguish between ghost authors and ghostwriters, ${ }^{2}$ the distinction between ghost authorship and ghostwriting used in this manuscript is consistent with those used in the crosssectional surveys conducted by Flanagin ${ }^{27}$ and Wislar ${ }^{20}$ and with the authorship criteria recommended by ICMJE. ${ }^{12}$ Distinguishing between these practices is important because it differentiates between undisclosed contributions that do and do not merit authorship. However, part of the reason for the disagreement about definitions of ghost authoring and ghostwriting stems from differences of opinion in the medical literature over how authorship is defined, particularly with regard to what constitutes 'substantial' contributions to a publication and the role of disclosure of contributions to a manuscript in the acknowledgments. ${ }^{67}$ The ICMJE criteria for authorship ${ }^{12}$ are the most well-recognised authorship criteria available, but leave much about authorship undefined. ${ }^{63} 68$ Despite this, the recent emphasis on author accountability in the ICMJE's fourth criterion is an attempt to better define authors as those persons who are responsible and accountable for the content of a published work. While a professional medical writer can take responsibility for the writing and how research is reported in a manuscript, the 
professional medical writer cannot take responsibility for the integrity of the research or be accountable for the clinical interpretation of the findings, unless he or she was involved in the generation of the research or its analysis. Responsibility for the integrity of research and accountability for the clinical interpretation of the findings is, and should always be, the responsibility of the authors. Until a more definitive model of authorship is universally accepted, the controversy and disagreement over definitions of authors, ghostwriters and ghost authors will remain. However, no matter what term is used, the practice of misleading readers about potential competing interests and hiding contributions to a published work, no matter whether these contributions should be most appropriately disclosed in the author byline or acknowledgements section, is unethical and totally unacceptable.

The strengths of this systematic review are that a broad search strategy was employed with few restrictions to minimise any potential for publication or language bias. All study designs and publication types were considered and there were no restrictions on language. Although the full text or abstracts of eight publications could not be retrieved, omission of these publications was unlikely to have biased the findings. Review of the abstracts or titles of these publications suggested that none reported numerical estimates of the prevalence of ghostwriting. The major finding of this review was the limitations of the reported evidence of ghostwriting in the medical literature. These limitations included the heterogeneity among the studies in the outcomes reported and populations investigated, the observational study designs and the retrospective nature of data collection. Given the nature of unethical authorship practices, it may not be feasible to conduct a prospective study on ghostwriting. However, based on the findings from this review, recommendations can be made to help researchers, authors, editors and peer-reviewers apply the same rigorous standards that are applied to the conduct and assessment of all clinical research and actively improve the quality of reporting of the evidence of unethical authorship practices.

Researchers should use a standard definition of ghostwriting so that the confusion around ghostwriters and ghost authors is not perpetuated. For example, the descriptive analysis of Danish industry-initiated trials used a non-standard definition of authorship, which is likely to have contributed to the very high prevalence of possible ghostwriting reported in this study (75\%). In this study, Gøtzsche $e t a l^{30}$ suggested that individuals who write the trial protocol, conduct the statistical analyses or who contribute to the writing of a publication should be included as authors. Indeed, the prevalence of ghost authorship in this study was $91 \%$ when the analyses included these individuals, irrespective of whether they had been appropriately acknowledged elsewhere. Given that the Gøtzsche et $a l^{30}$ study has been downloaded more than 3000 times since publication ${ }^{69}$ and cited repeatedly as evidence not only of ghost authorship but also of ghostwriting, ${ }^{40} 444648$ it would have been illuminating if the authors had included an estimate of the prevalence of ghostwriting using standard definitions for comparison. In addition to using standard definitions of ghostwriting, researchers and authors should ensure that cited evidence of ghostwriting is reported accurately without unwarranted generalisations. Publications based on personal commentary should be avoided and studies that use non-standard definitions, specific populations or that were conducted before a change in practice (eg, before the adoption of the Good Publication Practice guidelines for communicating company sponsored research in 2003 (GPP) $^{70}$ and in 2009 (GPP2) $^{11}$ ) should be described in context.

Editors and peer reviewers have a duty to prevent outdated, misleading or mistaken evidence on the prevalence of ghostwriting from being published and perpetuated. Close attention should be paid to the internal and external validity of the study, the definitions used, how the data are reported and whether the data are interpreted within the context of current practices. When assessing a submitted manuscript on ghostwriting, editors should consider using peer reviewers with expertise in the appropriate study designs (eg, survey research) and ethical publication practices. Collectively, these actions could help prevent further questionable evidence on the prevalence of ghostwriting from being published.

In conclusion, the findings from this systematic review showed that reports of the prevalence of ghostwriting in the medical literature are limited by the varied definitions used to describe unethical authorship practices, the types of study designs employed and the populations assessed. To improve reporting, researchers should not inflate estimates using non-standard definitions of ghostwriting nor conflate ghostwriting with other unethical authorship practices. While open and transparent debate should be encouraged, editors and peer reviewers should not accept articles that incorrectly cite or interpret primary publications that report the prevalence of ghostwriting.

Acknowledgements The author acknowledges and thanks Sericka McGee, PharmD, Hamilton House, for her contributions to the conception and design of the systematic literature review and the collection and screening of articles for inclusion. Sericka McGee contributed to an earlier version of the systematic review (search dates 1966 to May 2011), which was presented at the 8th Annual Meeting, International Society for Medical Publication Professionals, 23-25 Apr 2012 and at the 72nd Annual Conference, American Medical Writers Association, 4-6 October 2012, Sacramento, USA. The author thanks Professor Karen Woolley, PhD, CMPP (ProScribe-Envision Pharma Group) and Dr Cindy Hamilton, PharmD, ELS (Hamilton House) for their stimulating and valuable contributions to the interpretation of the findings from this study and for their critical review during the drafting of the manuscript.

Contributors SS contributed to the conception and design of the systematic literature review, the collection and screening of publications, and the analysis and interpretation of the findings. SS drafted and critically revised draft versions of the manuscript and gave final approval for submission.

Funding The author was supported (eg, reimbursement of expenses for conference travel) by her employer, ProScribe-Envision Pharma Group, for the submitted work. No other funding was received to conduct this research. 
Competing interests The author is a professional medical writer who is actively involved in national and international not-for-profit organisations that encourage ethical medical writing practices.

Provenance and peer review Not commissioned; externally peer reviewed.

Data sharing statement All data associated with the study are fully available to the author.

Open Access This is an Open Access article distributed in accordance with the Creative Commons Attribution Non Commercial (CC BY-NC 3.0) license, which permits others to distribute, remix, adapt, build upon this work noncommercially, and license their derivative works on different terms, provided the original work is properly cited and the use is non-commercial. See: http:// creativecommons.org/licenses/by-nc/3.0/

\section{REFERENCES}

1. Woolley KL, Gertel A, Hamilton CW, et al. Time to finger point or fix? An invitation to join ongoing efforts to promote ethical authorship and other good publication practices. Ann Pharmacother 2013:47:1084-7.

2. Scott-Lichter D; The Editorial Policy Committee of the Council of Science Editors. CSE's white paper on promoting integrity in scientific journal publications, 2012 update. Wheat Ridge, CO, 2012. http://www.councilscienceeditors.org/files/public/entire_whitepaper. pdf (accessed 30 Dec 2013).

3. Mathews AW. Ghost story: at medical journals, writers paid by industry play big role. Wall St $J$ (East Ed) 2005:A1-8.

4. Abbasi K. Editor's choice: transparency and trust. BMJ 2004;329:0.8

5. Collier R. Prevalence of ghostwriting spurs calls for transparency. Can Med Assoc J 2009;181:E161-2.

6. Healy D, Cattell D. Interface between authorship, industry and science in the domain of therapeutics. Br J Psychiatry 2003;183:22-7.

7. PLoS Med Editors. Ghostwriting: the dirty little secret of medical publishing that just got bigger. PLoS Med 2009;6:e1000156.

8. Ross JS, Hill KP, Egilman DS, et al. Guest authorship and ghostwriting in publications related to rofecoxib: a case study of industry documents from rofecoxib litigation. JAMA 2008;299:1800-12.

9. Gøtzsche PC, Kassirer JP, Woolley KL, et al. What should be done to tackle ghostwriting in the medical literature? PLoS Med 2009;6:e23.

10. Woolley KL, Ely JA, Woolley MJ, et al. Declaration of medical writing assistance in international peer-reviewed publications. JAMA 2006;296:932-4.

11. Graf C, Battisti WP, Bridges D, et al. Research methods \& reporting Good publication practice for communicating company sponsored medical research: the GPP2 guidelines. BMJ 2009;339:b4330.

12. International Committee of Medical Journal Editors. Recommendations for the conduct, reporting, editing and publication of scholarly work in medical journals. Roles and responsibilities of authors, contributors, reviewers, editors, publishers, and owners: defining the role of authors and contributors. 2013. http://www.icmje. org/roles_a.html (accessed 30 Dec 2013).

13. Nastasee S. Acknowledgement of medical writers in medical journal articles: a comparison from the years 2000 and 2007. AMWA J 2010;25:2-6.

14. World Association of Medical Editors. Policy Statement: Ghost writing initiated by commercial companies, Revised 15 June 2005. http:// www.wame.org/resources/policies\#ghost (accessed 30 Dec 2013).

15. Editors. Ghost writers in the sky. J Urol 2008:179:809-10.

16. Woolley KL. Goodbye Ghostwriters! How to work ethically and efficiently with professional medical writers. Chest 2006;130:921-3.

17. Jacobs A, Wager E. European Medical Writers Association (EMWA) guidelines on the role of medical writers in developing peer-reviewed publications. Curr Med Res Opin 2005;21:317-22.

18. Hamilton C. AMWA position statement on the contributions of medical writers to scientific publications. AMWA J 2003;18:13-16.

19. Bennett C, Khangura S, Brehaut J, et al. Reporting guidelines for survey research: an analysis of published guidance and reporting practices. PLoS Med 2011;8:e1001069.

20. Wislar JS, Flanagin A, Fontanarosa PB, et al. Honorary and ghost authorship in high impact biomedical journals: a cross sectional survey. BMJ 2011;343:d6128.

21. Vinther S, Rosenberg J. Appearance of ghost and gift authors in Ugeskrift for Laeger and Danish Medical Journal. Dan Med J 2012;59:1-6.
22. Price J, Dake J, Oden L. Authorship of health education articles: guests, ghosts and trends. Am J Health Behavior 2000;24:290-9.

23. Mowatt G, Shirran L, Grimshaw JM, et al. Prevalence of honorary and ghost authorship in Cochrane reviews. JAMA 2002;287:2769-71.

24. Mirzazadeh A, Navadeh S, Rokni M, et al. The prevalence of honorary and ghost authorships in Iranian bio-medical journals and its associated factors. Iran J Public Health 2011;40:15-21.

25. Jacobs A, Hamilton C. Decreased evidence of ghostwriting in a 2008 vs 2005 survey of medical writers. Write Stuff 2009;18:118-23.

26. Ghajarzadeh M. Guest authors in an Iranian journal. Dev World Bioeth 2014;14:15-19.

27. Flanagin A, Carey LA, Fontanarosa PB, et al. Prevalence of articles with honorary authors and ghost authors in peer- reviewed medical journals. JAMA 1998;280:222-4.

28. Dotson B, Slaughter RL. Prevalence of articles with honorary and ghost authors in three pharmacy journals. Am J Health Syst Pharm 2011;68:1730-4.

29. Suda KJ, Hurley AM, McKibbin T, et al. Publication of noninferiority clinical trials: changes over a 20 -year interval. Pharmacotherapy 2011;31:833-9.

30. Gøtzsche PC, Hróbjartsson A, Johansen HK, et al. Ghost authorship in industry-initiated randomised trials. PLoS Med 2007;4:0047-52.

31. Rees T, Kelly M, Smith S. How much do healthcare professionals know about GPP authorship criteria? Curr Med Res Opin 2013;29: S18-S9.

32. Hao X, Qian S, You S, et al. Ghost writers and honorary authorship: a survey from the Chinese Medical Journal [abstract]. Sixth International Congress on Peer Review and Biomedical Publication; 10-12 September 2009; Vancouver, BC, Canada 2009.

33. Hamilton CW, Jacobs A. Evidence of decreased ghostwriting in a series of three surveys of medical communicators conducted between 2005 and 2011. AMWA J 2012;27:115.

34. Elliott $C$. Pharma goes to the laundry: public relations and the business of medical education. Hastings Cent Rep 2004;34:18-23.

35. Moffatt B, Elliott C. Ghost marketing: pharmaceutical companies and ghostwritten journal articles. Perspect Biol Med 2007;50:18-31.

36. Scheife RT. A ghost in the machine. Pharmacotherapy 2009;29:363-4.

37. MacLennan A, Sturdee D, Fenton A, et al. Editorial: ghost writers, vested interest and funding disclosures. Climacteric 2010;13:301-2.

38. Nahai F. Ghostwriting: Stringent disclosure policies take aim at authorship abuses. Aesthet Surg J 2010;30:265-6.

39. Ngai S, Gold JL, Gill SS, et al. Haunted manuscripts: ghost authorship in the medical literature. Account Res 2005;12:103-14.

40. Bosch X. Exorcising ghostwriting. Ghostwriting could potentially have serious repercussions for science and should therefore be treated as research misconduct. EMBO Rep 2011:12:489-94.

41. Wiwanitkit V. Ghostwriting: an existing problem. Am J Med 2012;125:e17.

42. Krimsky S. When conflict-of-interest is a factor in scientific misconduct. Med Law 2007;26:447-63.

43. Langdon-Neuner E. Medical ghost-writing. Mens Sana Monogr 2008:6:257-73

44. Tharyan P. Evidence-based medicine: can the evidence be trusted? Indian J Med Ethics 2011;8:201-7.

45. Paul M. On transparency, responsibility, and accountability. Clin Microbiol Infect 2009;15:1100-2.

46. Bavdekar SB. Authorship issues. Lung India 2012;29:76-80.

47. Gorski A, Letkiewicz S. 'Medical writing' and ghostwriting as ethical challenges in medical communication. Transplant Proc 2010;42:3335-7.

48. Matías-Guiu J, García-Ramos R. Ghost-authors, improvement article communication, and medical publications. Neurologia 2011;26:257-61.

49. McHenry L. Of sophists and spin-doctors: industry-sponsored ghostwriting and the crisis of academic medicine. Mens Sana Monogr 2010;8:129-45.

50. Healy DT. Transparency and trust: figure for ghost written articles was misquoted. BMJ 2004;329:1345.

51. Mitrany D. The ghostwriting controversy: time for a proper burial. Science Editor 2005;28:187.

52. Kmietowicz Z. Consumer organisations criticise influence of drug companies. BMJ 2004;329:937.

53. Bonita RE, Adams S, Whellan DJ. Reporting of clinical trials: publication, authorship, and trial registration. Heart Fail Clin 2011;7:561-7.

54. Anon. Ghostwriting in medical literature. Int J Occup Environ Health 2011:17:168-77.

55. Anon. High prevalence of ghost authorship in industry-initiated trials. Nat Clin Pract Nephrol 2007;3:240. 
56. Baethge C. On ghost authorship and reviews: the 6th International Congress on Peer Review and Biomedical Publication. Deutsches Arzteblatt 2009;106:731-2.

57. Flanagin A. Research on authorship. What do we know about the prevalence of ghost authorship? Council of Science Editors Annual Meeting; May 14-18, Atlanta, Georgia, 2010.

58. Murray S, Brophy J, Palepu A. Open Medicine's ghost and guest authorship policy. Open Med 2010;4:e11-12.

59. Moore N. Transparency and trust: clear definition of ghost writing would be helpful. BMJ 2004;329:1345-6.

60. Hargreaves S. Industry funded trials often have ghost authorship. BMJ 2007;334:223

61. Jones N. Ghosts still present in the medical machine. Nature 2009;461:325.

62. Williams M. An inquiry by the UK House of Commons Health Select Committee into the influence of the pharmaceutical industry (18 October). 2004. http://www.meactionuk.org.uk/HoC_Select Ctte_Inquiry_into_Pharma.htm (accessed $30 \mathrm{Dec} 2013$ ).

63. Marušić $A$, Bošnjak $L$, Jeronšić $A$. A systematic review of research on the meaning, ethics and practices of authorship across scholarly disciplines. PLOS ONE 2011;6:e23477.
64. Bailey M. Science editing and its effect on manuscript acceptance time. AMWA J 2011;26:147-52.

65. Jacobs A. Adherence to the CONSORT guideline in papers written by professional medical writers. Write Stuff 2010; 19:196-200

66. Woolley KL, Lew RA, Stretton S, et al. Lack of involvement of medical writers and the pharmaceutical industry in publications retracted for misconduct: a systematic, controlled, retrospective study. Curr Med Res Opin 2011;27:1175-82.

67. Leo L, Lacasse J. Medical ghostwriting: a university sanctioned sleight of hand? Society 2012;49:310-12.

68. Malički M, Jerončić A, Marušić M, et al. Why do you think you should be the author on this manuscript? Analysis of open-ended responses of authors in a general medical journal. BMC Med Res Methodol 2012;12:189.

69. PLoS Med. Article metrics: ghost authorship in industry-initiated randomised trials. PLoS Med 2014. http://www.plosmedicine.org/ article/metrics/info\%3Adoi\%2F10.1371\%2Fjournal.pmed.0040019 (accessed 9 Jul 2014).

70. Wager E, Field EA, Grossman L. Good publication practice for pharmaceutical companies. Curr Med Res Opin 2003;19:149-54. 